\title{
ON POSITIVE SOLUTIONS OF SEMILINEAR ELLIPTIC EQUATIONS
}

\author{
E. N. DANCER AND KLAUS SCHMITT
}

(Communicated by Walter Littman)

\begin{abstract}
This paper is concerned with necessary conditions for the existence of positive solutions of the semilinear problem $\Delta u+f(u)=0, x \in \Omega, u=0, x \in \partial \Omega$, whose supremum norm bears a certain relationship to zeros of the nonlinearity $f$. We first discuss the smooth case (i.e., $f$ and $\partial \Omega$ smooth) and then show how to obtain similar results in the nonsmooth case.
\end{abstract}

1. Introduction. Consider the boundary value problem

$$
\begin{cases}\Delta u+\lambda f(u)=0, & x \in \Omega, \\ u=0, & x \in \partial \Omega,\end{cases}
$$

where $\Omega$ is a bounded domain in $\mathbf{R}^{n}, n>1$, with smooth boundary, $f$ is a $C^{1}$ nonlinearity as depicted in Figure 1 , and $\lambda>0$.

In $[\mathbf{H}]$, Hess showed that if

$$
\int_{s_{0}}^{s_{2}} f(s) d s>0
$$

then for all $\lambda \gg 1(1)$ has a solution $u(x)$ satisfying

$$
\left\{\begin{array}{l}
u_{\max }=\max _{x \in \Omega} u(x) \in\left(s_{1}, s_{2}\right), \\
u(x)>0, \quad x \in \Omega .
\end{array}\right.
$$

If the domain $\Omega$ satisfied a certain symmetry condition, it was shown in $[\mathbf{C o S}]$ that in fact

$$
u_{\text {max }}>r \text {, }
$$

for any solution $u$ satisfying (3) where $r \in\left(s_{1}, s_{2}\right)$, is given by

$$
\int_{s_{1}}^{r} f(s) d s=0 .
$$

The questions to be addressed in this note are whether (2) is in fact a necessary condition for the existence of solutions of (1) satisfying (3) and whether (4) holds for arbitrary domains. We shall answer these questions in the affirmative employing sub- and super-solution techniques and a result of Gidas, Ni and Nirenberg [GNN] about the radial symmetry of positive solutions of (1) if $\Omega$ is a ball. We shall also

Received by the editors August 7, 1986.

1980 Mathematics Subject Classification (1985 Revision). Primary 35J65, 35J25, 35B45.

Research supported by NSF Grant DMS 8501311. 


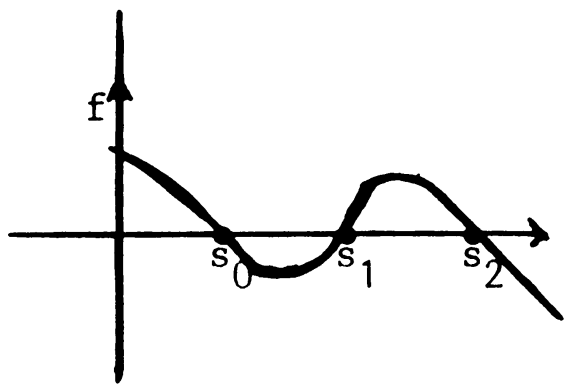

FIGURE 1

observe that the smoothness requirements upon $f$ and $\Omega$ may be considerably relaxed, that $f(0)$ need not be nonnegative and we shall give some applications which improve results of the first author in [D1, D2] for systems and answer a question of DeFigueiredo [DF]. We point out that Clement and Sweers [CS] have independently established the necessity of (2) by techniques different from ours. Their method, however, seems to require more regularity of $f$. Since the parameter $\lambda$ does not play a role in our considerations we shall replace $\lambda f$ by $f$ henceforth.

The second author acknowledges many interesting conversations with Professor Klaus Kirchgässner concerning the topic of this paper.

2. Necessary conditions for existence. Let us assume that $f: \mathbf{R} \rightarrow \mathbf{R}$ is a $C^{1}$ function and let the following conditions hold: There exist $0 \leqslant s_{0}<s_{1}<s_{2}$ such that

$$
\left\{\begin{array}{l}
f\left(s_{i}\right)=0, \quad i=1,2, \\
f\left(s_{0}\right) \leqslant 0, \\
f(s)<0, \quad s_{0}<s<s_{1}, \\
f(s)>0, \quad s_{1}<s<s_{2}
\end{array}\right.
$$

and let

$$
\int_{s_{0}}^{s_{2}} f(s) d s \leqslant 0 .
$$

We then have the following theorem.

THEOREM 1. Assume that $f$ satisfies (6) and (7) and let $\Omega$ be a bounded domain with smooth boundary. If (1) has a positive solution $u, u$ cannot satisfy (3).

Proof. Assume (1) has a positive solution $u$ which satisfies (3). We assume first that $f(0) \geqslant 0$, this restriction will be removed later.

Let $B$ denote a ball in $\mathbf{R}^{n}$, centered at the origin such that $\bar{\Omega} \subset B$. We consider the boundary value problem

$$
\begin{cases}\Delta u+f(u)=0, & x \in B, \\ u=0, & x \in \partial B .\end{cases}
$$


Let $\alpha(x)$ be defined by

$$
\alpha(x)= \begin{cases}u(x), & x \in \bar{\Omega}, \\ 0, & x \in \bar{B} \backslash \Omega .\end{cases}
$$

Then $\alpha$ is a sub-solution of (8) (see [BL]) and $\beta(x) \equiv s_{2}$ is a super-solution. Hence (see $[\mathbf{S}])(8)$ has a solution $v(x)$ such that

$$
\alpha(x) \leqslant v(x) \leqslant \beta(x), \quad x \in \bar{\Omega},
$$

i.e., (8) has a positive solution $v$ such that $v_{\max } \in\left(s_{1}, s_{2}\right)$. (The maximum principle ensures that $v_{\max }=s_{2}$ is impossible.)

It follows from the symmetry results of [GNN] that $v$ is radially symmetric and $v$ has a unique maximum at $x=0$. Hence $v$ is a positive solution of the ordinary differential equation

$$
\left\{\begin{array}{l}
v^{\prime \prime}+(n-1) v^{\prime} / r+f(v)=0, \\
v^{\prime}(0)=0=v(R),
\end{array}\right.
$$

where $R$ is the radius of $B$, and $v^{\prime}<0,0<r<R$. Integrating (9), we obtain

$$
\frac{1}{2}\left[v^{\prime}(r)\right]^{2}+\int_{v(0)}^{v(r)} f(s) d s=-(n-1) \int_{0}^{r} \frac{\left[v^{\prime}(s)\right]^{2}}{s} d s .
$$

Choosing $r$ so that $v(r)=s_{0}$, we get

$$
\frac{1}{2}\left[v^{\prime}(r)\right]^{2}+\int_{v_{\max }}^{s_{0}} f(s) d s<0 .
$$

On the other hand, because of (7) we get

$$
\int_{s_{0}}^{v_{\max }} f(s) d s \leqslant \int_{s_{0}}^{s_{2}} f(s) d s \leqslant 0
$$

contradicting (10).

We note that the assumption that $f(0) \geqslant 0$ was needed in order to conclude that $\alpha(x)$ is a lower solution of (8).

Next assume that $f(0)<0$. Again assume that (1) has a positive solution $v$ satisfying (3). Define $\tilde{f}$ so that

$$
\begin{aligned}
& \tilde{f}(s) \geqslant f(s), \quad 0 \leqslant s \leqslant v_{\max }, \\
& \tilde{f}(0) \geqslant 0, \quad \int_{s_{0}}^{s_{2}} \tilde{f}(s) d s<0 .
\end{aligned}
$$

Here we use that $v_{\max }<s_{2}$. Then

$$
\Delta v+\tilde{f}(v) \geqslant \Delta v+f(v)=0 .
$$

Hence $v$ is a sub-solution of

$$
\begin{cases}\Delta u+\tilde{f}(u)=0, & x \in \Omega \\ u=0, & x \in \partial \Omega\end{cases}
$$

and as before $\beta(x) \equiv s_{2}$ is a super-solution. Hence (11) has a solution $u$ satisfying $v(x) \leqslant u(x) \leqslant s_{2}$, i.e., $u$ satisfies (3). We now proceed as in the first part of the proof with $\tilde{f}$ in place of $f$. 
3. Lower bounds on $u_{\max }$. Let $f$ be as in the previous section and let $u$ be a positive solution of (1) satisfying (3); then it follows from Theorem 1 that (2) holds.

THEOREM 2. Let $u$ be a positive solution of (1) satisfying (3). Let $r$ be defined by (5). Then $u_{\max } \geqslant r$.

Proof. Assume $u_{\max }<r$. Let $\tilde{f}$ be defined as follows:

$$
\tilde{f}(s)= \begin{cases}f(s), & 0 \leqslant s \leqslant u_{\max }, \\ g(s), & u_{\max }<s,\end{cases}
$$

where $g(s)$ is chosen such that $\tilde{f}(s)>0, s_{1}<s<s_{2}, \tilde{f}\left(s_{2}\right)=0$, and $\int_{s_{0}}^{s_{2}} \tilde{f}(s) d s \leqslant 0$. This clearly can be done since $\int_{s_{0}}^{u_{\max }} f(s) d s<0$. Note that $u$ also solves the problem

$$
\begin{cases}\Delta u+\tilde{f}(u)=0, & x \in \Omega, \\ u=0, & x \in \partial \Omega .\end{cases}
$$

According to Theorem 1, however, this problem cannot have a positive solution satisfying (3).

We note that this result generalizes the major result of $[\mathbf{C o S}]$, when $\Omega$ was required to satisfy certain symmetry conditions.

4. Necessary conditions - the case of unbounded domains. Let $\Omega$ be a domain in $\mathbf{R}^{n}$ where $n>1$ with smooth boundary such that $\Omega$ lies on one side of some given hyperplane. Without loss in generality we may then assume that $\bar{\Omega} \subseteq\left\{x \in \mathbf{R}^{n}\right.$ : $\left.x_{1}>0\right\}$. The purpose of this section is to demonstrate the validity of Theorem 1 for such domains. Let us denote by $T_{1}$ the open half-space $T_{1}=\left\{x \in \mathbf{R}^{n}: x_{1}>0\right\}$. We first consider the problem

$$
\begin{cases}\Delta u+f(u)=0, & x \in T_{1}, \\ u=0, & x \in \partial T_{1},\end{cases}
$$

and prove the following lemma.

Lemma 3. Let $f$ be as in Theorem 1 , except that $s_{0}>0$ or $f(0) \geqslant 0$ or $\int_{s_{0}}^{s_{2}} f(s) d s<0$. Then (12) has no nonnegative solutions $u$ with $\|u\|_{\infty} \in\left(s_{1}, s_{2}\right]$.

Proof. Let us assume that (12) has such a solution $z$. Then $z$ is, of course, a subsolution of (12) and $\beta(x) \equiv s_{2}$ is a super-solution. As in the case of bounded domains the problem (12) will have a maximal solution $w$, with $z(x) \leqslant w(x) \leqslant \beta(x)$, $x \in \bar{T}_{1}$. (The proof of this fact follows in much the same way as that for bounded domains, see e.g. $[\mathbf{N}, \mathbf{O}]$. Note that $w$ is also the maximal solution with $0 \leqslant w(x) \leqslant$ $\beta(x)$.)

Let us write $x=\left(x_{1}, y\right), x_{1} \in \mathbf{R}, y \in \mathbf{R}^{n-1}$. Then for any fixed $\hat{y} \in \mathbf{R}^{n-1}$, the function $w\left(x_{1}, y+\hat{y}\right)$ is also a solution of (12) between 0 and $s_{2}$, and hence it easily follows from maximality that in fact $w$ will only depend upon $x_{1}$. Thus $w$ satisfies

$$
\left\{\begin{array}{l}
w^{\prime \prime}+f(w)=0 \\
w(0)=0, \quad w\left(x_{1}\right)>0,0<x_{1}<\infty, \\
\sup w \in\left(s_{1}, s_{2}\right] .
\end{array}\right.
$$

Hence, integrating (13) and using (7) we obtain a contradiction. 
THEOREM 4. Let $\Omega$ be a domain with smooth boundary lying on one side of a hyperplane, and let $f$ be as in Theorem 1 , except that $s_{0}>0$ or $f(0) \geqslant 0$ or $\int_{s_{0}}^{s_{2}} f(s) d s<0$. Then (3) has no nonnegative solution $u$ with $\|u\|_{\infty} \in\left(s_{1}, s_{2}\right]$.

Proof. We may assume that $\bar{\Omega} \subset T_{1}$. If (3) has such a solution, then

$$
\alpha(x)= \begin{cases}u(x), & x \in \Omega, \\ 0, & x \notin \Omega,\end{cases}
$$

is a sub-solution of (12) and $s_{2}$ is a super-solution of that problem. Hence (12) has a nonnegative solution $v$ with $\|v\|_{\infty} \in\left(s_{1}, s_{2}\right]$, contradicting Lemma 3. If $f(0)<0$, we must modify $f$ as in the proof of Theorem 1 .

REMARK. Observe that Lemma 3 could equally well have been used to establish Theorem 1.

REMARK. The proof of Theorem 4 shows that the result is still true without the extra assumptions if $\|u\|_{\infty}<s_{2}$.

REMARK. It is possible to construct examples which show that Theorem 4 fails if $n \geqslant 2$ and $\Omega$ is the exterior of a ball.

5. Some applications. Consider the boundary value problem

$$
\begin{cases}\Delta u+\lambda \sin u=0, & x \in \Omega, \\ u=0, & x \in \partial \Omega\end{cases}
$$

where $\Omega$ is a bounded domain with smooth boundary. It is easy and classical to show that (14) has a unique positive solution $u$ with $u_{\max }<\pi$ for any $\lambda>\lambda_{1}$ where $\lambda_{1}$ is the principal eigenvalue of $-\Delta$ with respect to zero Dirichlet boundary data on $\partial \Omega$. To see that there are no other positive solutions we apply Theorem 1 with $s_{0}=(2 n-1) \pi, s_{1}=2 n \pi, s_{2}=(2 n+1) \pi$. This answers a question posed in [DF].

We next consider examples from [D1 and D2]. Let $a, b, c, g, e, f$, and $\tau$ be positive constants, let $k(s)$ be given by

$$
k(s)=a-b s-\frac{c}{g}(e-f s)^{+},
$$

and assume that $k(0) \leqslant 0, a>b e / f$, and $\int_{0}^{a / b} s k(s) d s \leqslant 0$. Using Theorem 1 we may conclude that the problem

$$
\begin{aligned}
& \Delta u+\tau u k(u)=0, \quad x \in \Omega, \\
& u=0, \quad x \in \partial \Omega,
\end{aligned}
$$

has no positive solution, and hence using the theory developed in [D2] we may conclude that the system

$$
\begin{aligned}
& \begin{aligned}
\Delta u+u(a-b u-c v) & =0, \quad x \in \Omega, \\
d \Delta v+v(e-f u-g v) & =0,
\end{aligned} \\
& u=v=0, \quad x \in \partial \Omega,
\end{aligned}
$$

has no strictly positive solution for small $d$. (A strictly positive solution is one where both $u$ and $v$ are nonnegative and neither vanishes identically in $\Omega$.) 
Let $a, b, d, m$, and $\gamma$ be positive constants with $1 \geqslant b \geqslant 1 / 2$, and consider the system

$$
\begin{array}{rlrl}
\Delta u+u[a(1-u)(u-b)-v] & =0, & & \\
d \Delta v+v[-v+m(u-\gamma)] & =0, & & \\
u=v & =0, & x \in \partial \Omega .
\end{array}
$$

If this system has a strictly positive solution $\left(u^{*}, v^{*}\right)$ (where the definition of strict positivity appears above), then it follows from the maximum principle that $u^{*}(x) \leqslant$ $1, x \in \bar{\Omega}$. Hence $u^{*}$ will be a sub-solution for the problem

$$
\begin{cases}\Delta u+u[a(1-u)(u-b)]=0, & x \in \Omega, \\ u=0, & x \in \partial \Omega .\end{cases}
$$

Since 1 is a super-solution, (15) will have a positive solution $u$ with $1 / 2 \leqslant u_{\max } \leqslant 1$. This together with the fact that $1 \geqslant b \geqslant 1 / 2$, contradicts Theorem 1 . Thus there is no strictly positive solution of (15).

6. Generalizations. In this section we considerably weaken our hypotheses by relaxing the conditions on the sign of $f$, on the smoothness of $f$ and on the smoothness of $\partial \Omega$.

Concerning the sign of $f$. The assumption that $f(s)<0$ on $\left(s_{0}, s_{1}\right)$ is not needed in Theorems 1 and 4. The proof remains the same. This will yield better results for certain $f$ having several humps. For functions $f$ as in Figure 2, it may happen that

$$
\int_{s_{0}}^{s_{2}} f(s) d s \leqslant 0, \quad \text { while } \int_{\bar{s}}^{s_{2}} f(s) d s>0 .
$$

Thus the generalized result implies in this case that there are no positive solutions $u$ with $u_{\max } \in\left(s_{1}, s_{2}\right)$. Note that we cannot apply Theorem 1 with $s_{0}$ replaced by $\bar{s}$. In particular, it follows that, in Hess $[\mathbf{H}]$, his sufficient conditions for the existence of solutions are all necessary conditions.

Concerning the smoothness of $f$. Suppose that $f$ is continuous and we look for solutions $u \in W^{2, p}(\Omega)$ for all $p \geqslant 1$, rather than $C^{2}$ solutions. In this case, Theorems 1 and 2 still hold. However, the proofs need some modification. To prove Theorem 1 we proceed again indirectly. Using a modification of $f$, if necessary, we may in fact assume that $\int_{s_{0}}^{s_{2}} f(s) d s<0$. We now use the theory of sub- and

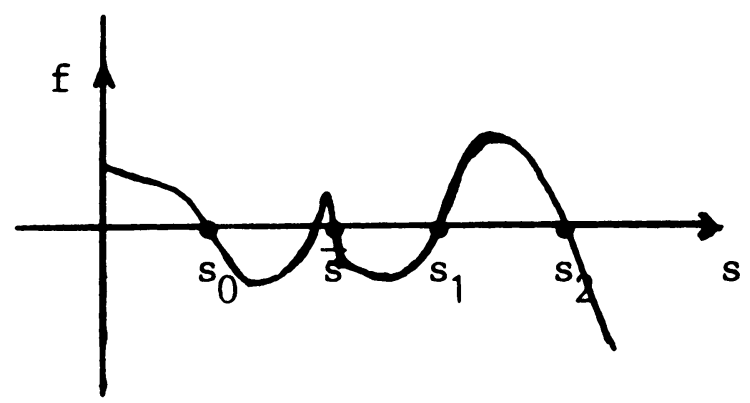

Figure 2 
super-solutions as in [DH] to find a solution $v$ of (1) on $B$ such that $\alpha(x) \leqslant v(x) \leqslant s_{2}$ on $B$. Now (we shall return to this point later), there must exist a maximal solution $\tilde{v}$ between the sub-solution 0 and the super-solution $s_{2}$. The existence of $v$ ensures that $\tilde{v}$ is nontrivial. By using rotations (cf. the proof of Lemma 3 ), the maximality implies that $\tilde{v}$ is radially symmetric. We now complete the proof as before. We note that it may be that $u_{\max }=s_{2}$. Indeed, our argument shows that there is no positive solution with $\|u\|_{\infty}=s_{2}$, if $f(0) \geqslant 0$ or $s_{0}>0$ or $\int_{s_{0}}^{s_{2}} f(s) d s<0$. We return to the question of the existence of a maximal solution; this seems known but we are unable to locate a reference. The existence of $\tilde{v}$ easily follows from the sub-super-solution result, compactness, and Zorn's Lemma provided we show that $w=\sup \left\{w_{1}, w_{2}\right\}$ is a sub-solution in a weak sense, whenever $w_{1}$ and $w_{2}$ are solutions. (Note that $w \in \dot{W}_{0}^{1.2}(B)$.) To show this, it suffices to prove that, for each $\varepsilon>0$ and for each $\phi \in C_{0}^{\infty}(B)$, with $\phi \geqslant 0$,

$$
\int_{\Omega} \nabla w \cdot \nabla \phi d x \leqslant \int_{\Omega}(f(w)+\varepsilon) \phi d x .
$$

Using a partition of unity argument, we see further that it suffices to show that for each $\varepsilon>0$ and each $x_{0} \in B$ there exists a neighborhood $\Omega_{x_{0}}$ of $x_{0}$ such that (16) holds for all $\phi \geqslant 0$ with support in $\Omega_{x_{0}}$. If $w_{1}\left(x_{0}\right) \neq w_{2}\left(x_{0}\right)$ we choose a neighborhood $\Omega_{x_{0}}$ such that $w_{1}(x)-w_{2}(x)$ has fixed sign on $\Omega_{x_{0}}$ and the result follows. If $w_{1}\left(x_{0}\right)=w_{2}\left(x_{0}\right)$, we choose a neighborhood $\Omega_{x_{0}}$ such that $f(x, w(x))+\varepsilon \geqslant$ $f\left(x, w_{i}(x)\right)$ on $\Omega_{x_{0}}$ (by continuity). Since $w_{1}$ and $w_{2}$ are sub-solutions relative to the equation

$$
\Delta w+f(w)+\varepsilon=0 \quad \text { on } \Omega_{x_{0}}
$$

it follows from [KS, Theorem II.6.6] that $w=\sup \left\{w_{1}, w_{2}\right\}$ is also a sub-solution of (17) on $\Omega_{x_{0}}$. Theorem 4 also holds if we assume that $\|u\|_{\infty}<s_{2}$ or $\int_{s_{0}}^{s_{2}} f(s) d s<0$ or $f$ is Lipschitz continuous at $s_{0}$. (The extra assumptions are needed for the onedimensional problem.)

Concerning the smoothness of $\partial \Omega$. The smoothness requirements imposed on $\partial \Omega$ may be considerably relaxed. It suffices to assume that $\Omega$ is a domain of class $C$, i.e., that for each $x_{0} \in \partial \Omega, \bar{\Omega}$ is locally homeomorphic to a half-space of $\mathbf{R}^{n}$, and there exists a compact subset $K$ of $\partial \Omega$ with zero capacity in $B$ (or equivalently $\mathbf{R}^{n}$ ) (see [KS] for the definition of capacity) such that $\partial \Omega \backslash K$ is a $C^{2}$ manifold. This class seems to include most "reasonable" domains with corners even if the corners are "cusp-like." In this case a solution is assumed to belong to $\dot{W}_{0}^{1.2}(\Omega) \cap L^{\infty}(\Omega)$. (It follows that they belong to $W_{\mathrm{loc}}^{2, p}(\Omega)$.) To see that our results still hold in this more general situation we note that the smoothness of $\partial \Omega$ was only used to ensure that $\alpha(x)$ (as defined in the proof of Theorem 1) is a sub-solution on $B$. Since $u \in \dot{W}_{0}^{1,2}(\Omega), \alpha \in \dot{W}_{0}^{1,2}(B)$, we thus have to show that

$$
\int_{\Omega} \nabla \alpha \cdot \nabla \phi d x \leqslant \int_{\Omega} f(\alpha) \phi d x
$$

for all nonnegative $\phi \in \dot{W}_{0}^{1.2}(B)$, and by continuity it suffices to show (18) only for a dense subset of $\dot{W}_{0}^{1.2}(B)$ of such $\phi$. If $\phi$ is smooth and vanishes in a neighborhood of $K,(18)$ follows by the argument used in [BL]. (All the integrals vanish near the 
"bad" part of $\partial \Omega$.) Thus it suffices to prove that such functions are dense in the nonnegative functions in $\dot{W}_{0}^{1,2}(B)$. It is well known that the assumption of zero capacity ensures this (and in fact, the capacity being zero is the only time when the density property holds). One way to see this is to find $w_{n}$ such that $w_{n}$ is Lipschitz continuous on $B$, has compact support in $B$, and satisfies $\int_{B}\left|\nabla w_{n}\right|^{2} d x \leqslant 1 / n$, $w_{n}=1$ in a neighborhood of $K$, and $0 \leqslant w_{n} \leqslant 1$ on $B$. (We first find $w_{n}$ such that $w_{n} \geqslant 1$ on $K$ and then replace it by $g\left(\left(1+\delta_{n}\right) w_{n}\right)$, where $g(y)=y$ if $y \leqslant 1$ and $g(y)=1$ if $y \geqslant 1$.) It suffices to approximate functions $\phi$ which are smooth and zero near $\partial B$. Since it is easy to show that for such $\phi,\left(1-w_{n}\right) \phi \rightarrow \phi$ in $\dot{W}_{0}^{1,2}(B)$ as $n \rightarrow \infty$ the result follows. If we wish to make the approximation smooth we use a mollifier. This completes the proof.

We finally remark that if $f$ is Lipschitz continuous, it may be shown that Theorem 1 holds for an arbitrary domain $\Omega$ by using some of the ideas in [KS]. The idea is to use the usual iteration $\left\{u_{n}\right\}_{n=1}^{\infty}$ to obtain the maximal solution on $B$ (where $\Omega \subseteq B$ ) and show inductively that, if $u$ is a positive solution on $\Omega$, then $u_{n}(x) \geqslant u(x)$ on $\Omega$.

\section{REFERENCES}

[BL] H. Berestycki and P. L. Lions, Some applications of the method of sub-and super-solutions, Lecture Notes in Math., vol. 782, Springer-Verlag, Berlin and New York, 1980, pp. 16-41.

[CS] P. Clement and G. Sweers, Existence and multiplicity results for a semilinear eigenvalue problem, preprint, 1986.

[CoS] C. Cosner and K. Schmitt, A priori bounds for positive solutions of semilinear elliptic equations, Proc. Amer. Math. Soc. 95 (1985), 47-50.

[D1] E. N. Dancer, On positive solutions of some pairs of differential equations, Trans. Amer. Math. Soc. 284 (1984), 729-743.

[D2] _ On positive solutions of some pairs of differential equations, J. Differential Equations 60 (1985), 236-258.

[DF] D. DeFigueiredo, On the uniqueness of positive solutions of the Dirichlet problem $-\Delta u=\lambda \sin u$, Nonlinear Partial Differential Equations and Applications, vol. 7 (H. Brezis and J. L. Lions, eds.), Pitman, London, 1984, pp. 80-83.

[DH] J. Deuel and P. Hess, $A$ criterion for the existence of solutions of nonlinear elliptic boundary value problems, Proc. Roy. Soc. Edinburgh Sect. A 74 (1975), 49-54.

[GNN] B. Gidas, W. Ni, and L. Nirenberg, Symmetry and related properties via the maximum principle, Comm. Math. Phys. 68 (1979), 209-243.

[H] P. Hess, On multiple positive solutions of nonlinear elliptic equations, Comm. Partial Differential Equations 6 (1981), 951-961.

[KS] D. Kinderlehrer and G. Stampacchia, An introduction to variational inequalities, Academic Press, New York, 1980.

[N] E. S. Noussair. On semilinear elliptic boundary value problems in unbounded domains, J. Differential Equations 41 (1981), 334-348.

[O] A. Ogata, On existence and multiplicity theorems for semilinear elliptic equations in exterior domains, Funkcial. Ekvac. 27 (1984), 281-299.

[S] K. Schmitt, Boundary value problems for quasilinear second order elliptic equations, Nonlinear Anal. 2 (1978), 263-309.

Department of Mathematics, The University of New England, Armidale, New South Wales 2351, Australia

Department of Mathematics, University of Utah, Salt Lake City, Utah 84112 